\title{
Clinical Outcome of Patients with Familial Hypercholesterolemia and Coronary Artery Disease Undergoing Partial Ileal Bypass Surgery
}

\author{
Jaqueline Scholz Issa, Artur Garrido Jr, Sérgio Diogo Giannini, N eusa Forti, Jayme Diament, \\ Henrique W alter Pinotti
}

São Paulo, SP - Brazil

\begin{abstract}
Familial hypercholesterolemia is characterized by high serum levels of total cholesterol and LDL-cholesterol. It may be homozygous or heterozygous. In homozygous patients, LDL-cholesterol levels range from 500 to $1000 \mathrm{mg} /$ $d L$ and coronary artery disease is precocious, usually manifesting itself between the $2^{\text {nd }}$ and $3^{\text {rd }}$ decades of life. The diagnosis is often made by the presence of xanthoma tuberosum and tendinous xanthomas that appear between the $1^{\text {st }}$ and $2^{\text {nd }}$ decades of life. The use of high doses of statins or even unusual procedures (apheresis, partial ileal bypass surgery, liver transplantation, gene therapy), or both, is necessary for increasing survival and improving quality of life, because a reduction in cholesterol levels is essential for stabilizing the coronary artery disease and reducing xanthomas.

We report our experience with 3 patients with xanthomatous familial hypercholesterolemia and coronary artery disease, who underwent partial ileal bypass surgery. Their follow-up over the years (approximately 8 years) showed a mean $30 \%$ reduction in total cholesterol, with a significant reduction in the xanthomas and stabilization of the coronary artery disease.
\end{abstract}

Familial hypercholesterolemia is characterized by high serum levels of total cholesterol and LDL-cholesterol (lowdensity lipoproteins). This increase results from the absence of or reduction in the LDL-specific receptors described by Goldstein and Brown ${ }^{1}$ and is determined by dominant autosomal genetic inheritance. The heterozygous form is one of the most frequent metabolic disorders in the general population (1:500), but the homozygous form is rare $(1: 1,000,000)$.

In homozygous individuals, no expression of the receptors occurs, resulting in LDL-cholesterol (LDL-C)

Instituto do Coração do Hospital das Clínicas - FMUSP

Mailing address: Jaqueline Scholz Issa - Rua Dr. Estevam de Almeida 74/64 05014-000 - São Paulo, SP, Brazil.

English version by Stela Maris C. Gandour values above $500 \mathrm{mg} / \mathrm{dL}$. In heterozygous individuals, a reduction in the number of receptors or their partial dysfunction occurs and the LDL-C values are around 500mg/ $\mathrm{dL}$, but always above the $90^{\text {th }}$ percentile of the population. The clinical presentation of familial hypercholesterolemia is through tendinous xanthomas, mainly in the Achilles tendons, and in the extension facies of the joints of the fingers, elbows, knees, and toes. Sometimes, cutaneous xanthoma tuberosum may occur. These symptoms appear during adolescence in heterozygous individuals and between 4 and 6 years of age in homozygous individuals. The coronary atherosclerotic disease appears early, in homozygous individuals usually between the $1^{\text {st }}$ and the $2^{\text {nd }}$ decades of life and in heterozygous individuals between the $4^{\text {th }}$ and the $5^{\text {th }}$ decades of life. The coronary atherosclerotic disease is frequently fatal.

Considering the severity of the disease and the therapeutic difficulties (mainly socioeconomic) in treating it, we report our experience in treating this disease using partial ileal bypass surgery. This is a reversible surgical procedure in which about 2 meters of the terminal ileum are excluded resulting in a reduction in cholesterol absorption and consequent reduction $( \pm 30 \%)$ in cholesterolemia. We do not know of any other patients in our country treated surgically for familial hypercholesterolemia.

\section{Case report}

Case 1 - A 40-year-old white female born in and coming from Bahia state reported the appearance of cutaneous and tendinous tumors since the age of 10 years. She was diagnosed with hypercholesterolemia (mean values of total cholesterol were $900 \mathrm{mg} / \mathrm{dL}$ ) at the age of 20 years. Her family history showed the following: her father died at the age of 50 years due to an acute myocardial infarction; one of her sisters died suddenly at the age of 30 years; another sister (next case reported) has xanthomas and hypercholesterolemia; a brother has xanthomas and hypercholesterolemia; and two children 7 and 9 years of age have hypercholesterolemia but no xanthomas (total cholesterol of $300 \mathrm{mg}$ / 
dL). The patient reported symptoms of stable angina at the age of 25 years and unstable angina at the age of 32 years, when she was referred to our Institution. The patient had xanthelasma, exuberant xanthoma tuberosum and tendinous xanthomas (fig. 1 and 2). She underwent cinecoronariography that showed trivessel obstruction as follows: right coronary artery, $80 \%$; left main coronary artery, $50 \%$; anterior descending artery, multiple lesions and $100 \%$; circumflex artery, diffuse irregularities; and hypokinesia of the left ventricular anterior wall $(++/ 4+)$. The patient underwent myocardial revascularization comprising the following: anastomosis of the left mammary artery to the left marginal artery; anastomosis of the right mammary artery (free graft) to the anterior descending artery; anastomosis of the epigastric artery to the diagonal artery; and a saphenous vein bypass graft to the right coronary artery. After hospital discharge, lipid-lowering drugs were prescribed in adequate doses (simvastatin 40mg/day and probucol 750mg/day). Ninety days after medication use, her lipid profile was as follows: total cholesterol,463mg/dL; HDL-C, 16mg/dL; LDLC, $420 \mathrm{mg} / \mathrm{dL}$; and triglycerides, $136 \mathrm{mg} / \mathrm{dL}$. The patient still complained of angina on modhate exertion.

Due to the severity of the disease and maintenance of

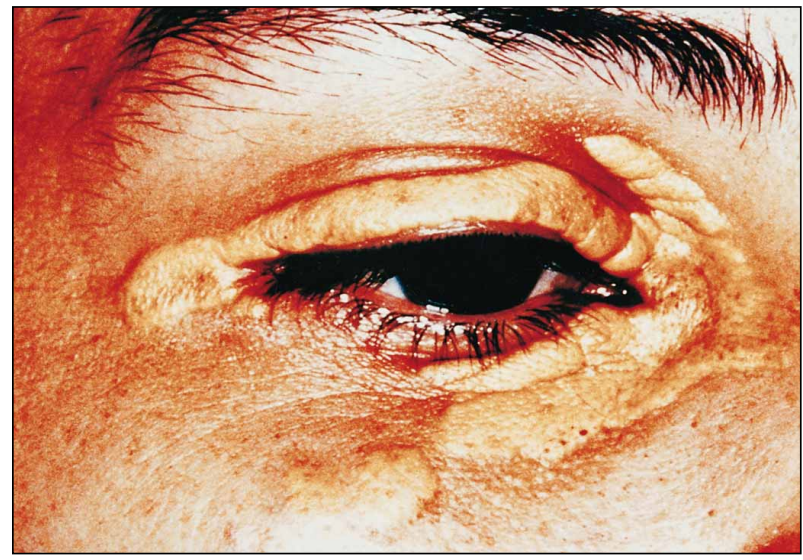

Fig. 1 - Xanthelasma - case 1.

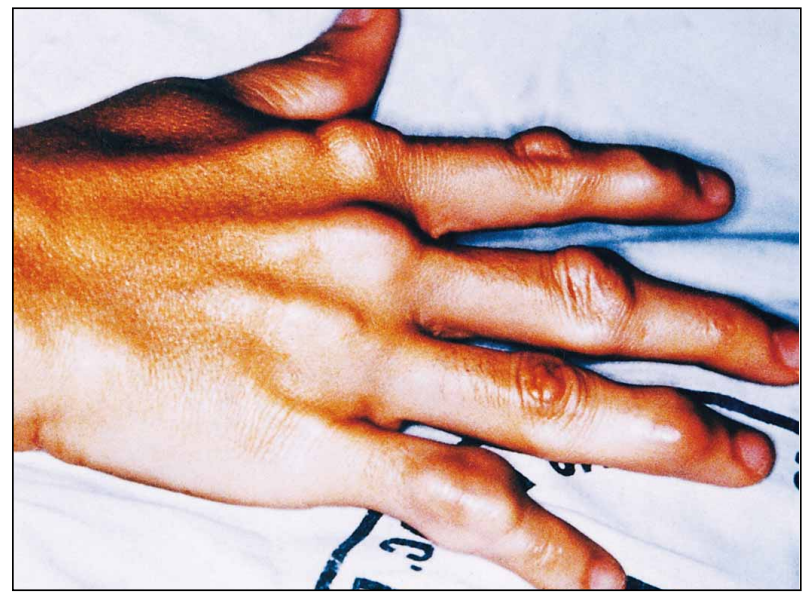

Fig. 2 - Tendinous xanthomas in the fingers in case 1, prior to ileal bypass surgery. high levels of cholesterolemia, the patient underwent an LDL-apheresis program that significantly reduced the lipid fractions (total cholesterol, 122mg/dL; HDL-C, 13mg/dL; LDL-C, $94 \mathrm{mg} / \mathrm{dL}$; triglycerides, $63 \mathrm{mg} / \mathrm{dL}$ ). Despite the efficacy of this procedure, the need for fortnightly sessions, the high cost, and the fact that the patient lived in another state did not allow the maintenance of this regimen.

The patient used lipid-lowering drugs (simvastatin $40 \mathrm{mg} /$ day and probucol $500 \mathrm{mg} /$ day) irregularly for one year: her total cholesterol reached values of $500 \mathrm{mg} / \mathrm{dL}$ with the medication, returning to basal values of $900 \mathrm{mg} / \mathrm{dL}$ without medication. Because of the economic difficulties of maintaining the medication, the persistence of high cholesterolemia due to the irregular use of the medication, and the angina on exertion, with a positive exercise test, partial ileal bypass surgery was proposed. The patient agreed to undergo the procedure and was operated upon on October 1991, when exclusion of 2 meters of the ileum and anastomosis between the proximal ileum and the cecum were performed.

A reduction in the xanthomas and xanthelasmas could be noticed one year after the procedure, and this reduction was even more significant 2 years after the procedure (figs, 3 and 4). The patient has been followed up for 8 years. Her symptoms of coronary heart disease have stabilized, and

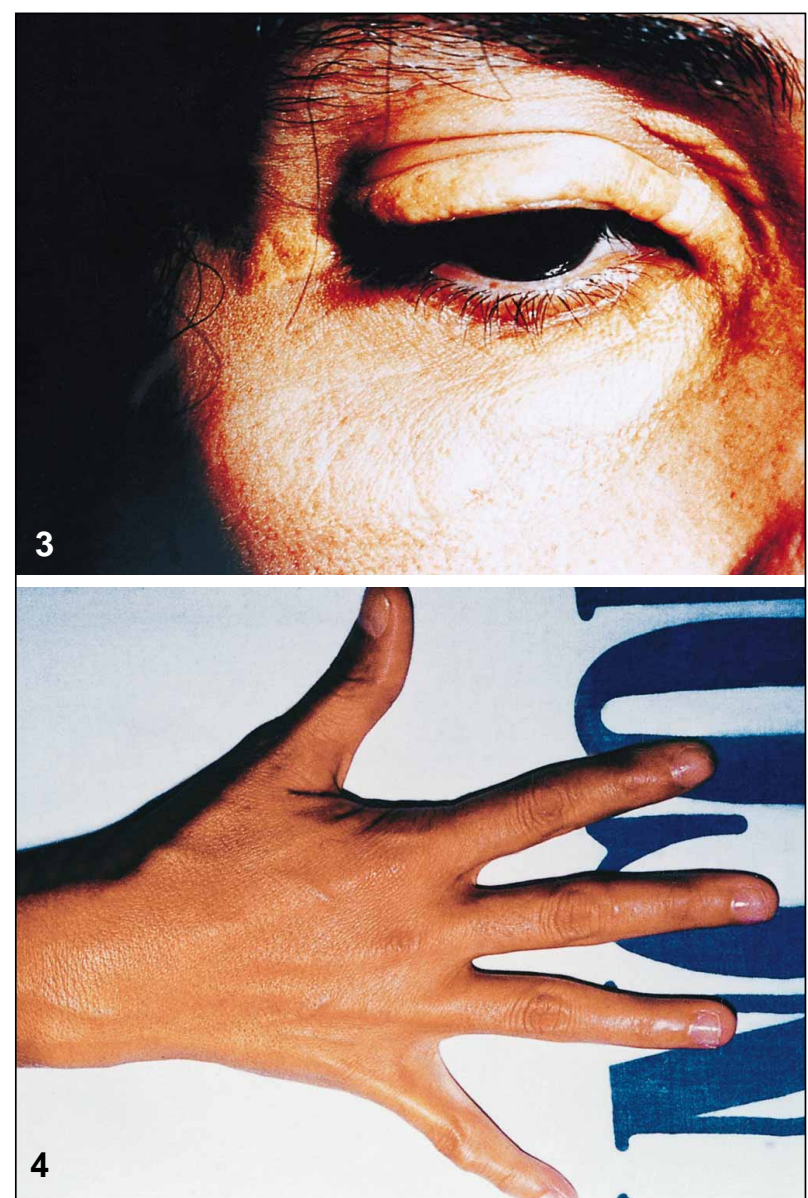

Figs. 3 and $4-$ Reduction in the xanthelasma and xanthomas, 2 years after ileal bypass surgery and irregular use of lipid-lowering drugs. 
she experiences angina only on great exertion. Her mean total cholesterol is around $580 \mathrm{mg} / \mathrm{dL}$ with no lipid-lowering drug. The use of $20 \mathrm{mg} /$ day of simvastatin reduced her total cholesterol to $450 \mathrm{mg} / \mathrm{dL}$. The LDL apheresis sessions were interrupted after the ileal bypass surgery.

Case 2 - A 37-year-old white female born in and coming from Bahia state reported a diagnosis of hypercholesterolemia at the age of 18 years (total cholesterol of $900 \mathrm{mg} /$ $\mathrm{dL}$ ) and appearance of xanthomas in the first decade of life. In 1990, she sought medical assistance at InCor at the age of 30 years, accompanied by her sister Case 1 . On clinical examination, she had exuberant xanthomas on her hands, elbows (fig. 5), and Achilles tendon. She did not report any symptom suggesting coronary atherosclerotic disease, but the exercise test showed signs of ischemia. The echocardiogram showed preserved ventricular function and an aortic valve gradient of $20 \mathrm{mmHg}$. The cineangiogram showed the following lesions: left main coronary artery, $30 \%$; anterior descending artery, $70 \%$; diagonal artery, $70 \%$; circumflex artery and right coronary artery, no irregularities; collateral flow from the right coronary artery to the anterior descending artery. The ventriculographic findings were normal, and no difference in pressure between the aorta and the left ventricle existed. Simvastatin (40mg/day) and probucol $(500 \mathrm{mg} /$ day $)$ were prescribed. The regular use of the medication resulted in the following lipid profile: total cholesterol, 578mg/dL; LDL-C, 508mg/dL; HDL-C, 20mg/dL; and triglycerides, $251 \mathrm{mg} / \mathrm{dL}$. Interruption of the medication caused an immediate return to basal values. Because of the same socioeconomic difficulties as those of the patient in case 1, in 1991, after the patient's consent, partial ileal bypass surgery was performed with exclusion of 2 meters of the ileum and ileocecal anastomosis.

The procedure resulted in a persistent reduction in the cholesterolemia with a total cholesterol of $580 \mathrm{mg} / \mathrm{dL}$. The patient became pregnant at the age of 35 years, and the pregnancy evolved uneventfully ${ }^{3}$. The newborn baby was eutrophic with hypercholesterolemia (total cholesterol of $300 \mathrm{mg} / \mathrm{dL}$ ). During pregnancy, the patient's cholesterol increased to $1,200 \mathrm{mg} / \mathrm{dL}$ after suspension of the medication. After the delivery, her total cholesterol dropped to $577 \mathrm{mg} / \mathrm{dL}$, HDL-C to $18 \mathrm{mg} / \mathrm{dL}$, LDL-C to $501 \mathrm{mg} / \mathrm{dL}$, and triglycerides to $290 \mathrm{mg} / \mathrm{dL}$, which are currently maintained with no lipid-lowering medication. The regular use of simvastatin $(20 \mathrm{mg} /$ day $)$ reduced the patient's total cholesterol to $470 \mathrm{mg} / \mathrm{dL}$.

During the follow-up, we observed a reduction in the xanthomas, and after a 6-year follow-up, the reduction in the tendinous xanthomas and in the xanthoma tuberosum of the elbow was more evident (fig. 6). In her last evaluation (8-year follow-up), the patient was asymptomatic. The exercise test did not show an ischemic response, the ventricular function remained normal on the echocardiogram, and an increase of the gradient of aortic stenosis $(49 \mathrm{mmHg})$.

Case 3-A 42-year-old black male born in and coming

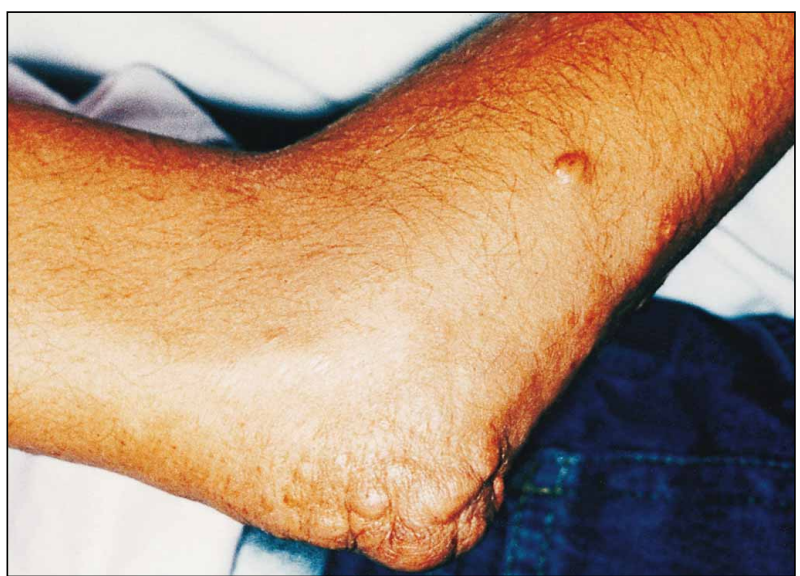

Fig. 5 - Tendinous xanthomas and xanthoma tuberosum in the elbow in case 2, prior to ileal bypass surgery.

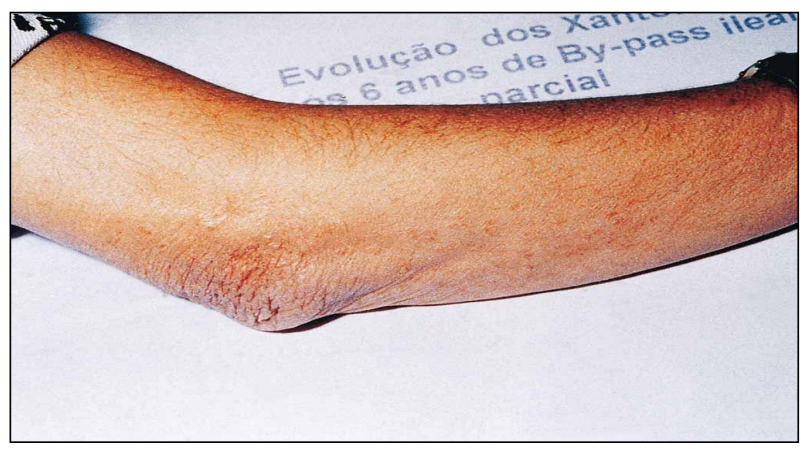

Fig. 6 - Reduction in the xanthomas (photograph obtained 6 years after the procedure and irregular use of lipid-lowering drugs).

from Rio de Janeiro reported that since the age of 20 years, an arcus cornealis had been noticed, as had xanthomas in the Achilles tendons, hands, and elbows, in addition to hypercholesterolemia $(550 \mathrm{mg} / \mathrm{dL})$. In his family history, the following were notable: his father had died of an acute myocardial infarction at the age of 50 years, and of 8 siblings, 2 had xanthomas and hypercholesterolemia. In 1991, the patient, then 35 years old, had an acute myocardial infarction in the inferior wall and underwent to a cinecoronarygraphy that revealed the following obstructions: right coronary artery, $100 \%$; anterior descending artery, some irregularities; diagonal artery, $90 \%$; circumflex artery with aneurysmal dilation; and marginal artery, $100 \%$. The ventriculography showed hypokinesia of the inferior wall. In the same year, the patient underwent myocardial revascularization with a mammary artery graft to the anterior descending artery, and saphenous vein bypass grafts to the right coronary artery and to the marginal artery. The patient was discharged with a prescription of $40 \mathrm{mg} /$ day of lovastatin. One year after regularly using the lipid-lowering drug, the patient's lipid profile was as follows: total cholesterol, $471 \mathrm{mg} / \mathrm{dL}$; LDL-C, $421 \mathrm{mg} / \mathrm{dL} ; \mathrm{HDL}-\mathrm{C}, 47 \mathrm{mg} / \mathrm{dL}$; and triglycerides, $59 \mathrm{mg} / \mathrm{dL}$. He reported then angina on great exertion. The association of $20 \mathrm{mg} /$ day of lovastatin and $8 \mathrm{~g} /$ day of cholestyramine reduced his total cholesterol to $322 \mathrm{mg} / \mathrm{dL}$ and his LDL-C to 
$244 \mathrm{mg} / \mathrm{dL}$. Because of socioeconomic difficulties in maintaining this medication and after formal consent, the patient underwent partial ileal bypass surgery in 1992. In the first 2 years following the procedure, the patient reported episodes of diarrhea after ingestion of a lipid-rich diet. This symptom was controlled with a reduction in the lipid content of the diet and use of cholestyramine (4 g/ day). After a 7-year follow-up, the patient is clinically well, with no significant episodes of diarrhea, maintenance of the body weight, and reduction in the xanthomas. A coronary angiography performed in 1996 showed no progression of the atherosclerotic plaques in the native bed, permeability of the saphenous vein bypass grafts, and occlusion of the mammary artery graft due to flow competition. The patient's total cholesterol level is $280 \mathrm{mg} / \mathrm{dL}$ and LDL-C is $198 \mathrm{mg} / \mathrm{dL}$. The use of $20 \mathrm{mg} /$ day of simvastatin allowed a reduction in total cholesterol to $216 \mathrm{mg} / \mathrm{dL}$ and in LDL-C to $140 \mathrm{mg} / \mathrm{dL}$.

\section{Discussion}

Isolated dietary changes are ineffective in reducing cholesterolemia in patients with familial hypercholesterolemia; therefore, the use of lipid-lowering drugs is mandatory. Among them, 3-hydroxy-3-methyl-glutaryl-coenzyme A (HMG-CoA) reductase inhibitors (statins) deserve special mention. Statins may be prescribed alone or in combination with other lipid-lowering drugs. For a significant reduction (greater than 30\%) in cholesterol, however, these drugs have to be used in high doses and continuously, making the long-term treatment very costly for the less fortunate portions of the population.

Considering the high morbidity and mortality of early coronary artery disease in patients with familial hypercholesterolemia and the difficulties of the medicamentous treatment (high cost), unusual procedures for treating hypercholesterolemia have been proposed in severe cases. Among them are partial ileal bypass surgery, apheresis, and gene therapy. Apheresis ${ }^{3}$ has proved to be a safe procedure capable of immediately and drastically reducing LDL-C in up to $70 \%$ of its value, but it requires repetition of the sessions at regular intervals of 15 to 21 days. Its application, therefore, is limited by its high cost and technological sophistication. Gene therapy may be the future option of treatment, but it is now in an experimental but progressive phase.

Partial ileal bypass surgery is a procedure that allows a permanent reduction in cholesterolemia. The technique developed and reported by Henry Buchwald ${ }^{4}$ in 1964 consists of the exclusion of $200 \mathrm{~cm}$ or $2 / 3$ of the small intestine (depending on which is greater) in its distal portion, by means of a blind loop, and termino-lateral anastomosis of the proximal segment in the region of the ileocecal valve. This procedure may be reversed. The exclusion of the ileal segment allows a significant reduction in the absorption of the cholesterol contained in the food, and it also interferes with the enterohepatic cycle of cholesterol. Therefore, it causes a $25 \%$ to $30 \%$ reduction in cholesterolemia and a
$30 \%$ to $35 \%$ reduction in LDL-C. It may have side effects, such as diarrhea, nephrolithiasis, cholelithiasis, weight loss, and, exceptionally, intestinal obstruction.

Aiming to assess the hypothesis that the reduction in cholesterol induced by partial ileal bypass surgery favors the reduction in morbidity and mortality due to coronary atherosclerotic disease in patients after myocardial infarction, a randomized study known as $\mathrm{POSCH}$ (Program on the Surgical Control of the Hyperlipidemias) $)^{5}$ was carried out. The population of the study consisted of 838 patients (males and females), 417 of whom comprised the control group that received only an AHA phase II diet. The remaining 421 patients underwent ileal bypass surgery. The authors concluded that the lipid changes caused by the partial ileal bypass surgery (reduction of $23.3 \%$ in total cholesterol and of $36.1 \%$ in LDL-C) accounted for slowing the progression of the coronary atherosclerotic disease in this group, with a smaller number of reinfarctions, angioplasty, and myocardial revascularization. The long-term follow-up, five years of observation after concluding the study ${ }^{6}$, with a mean time of patient observation of 12 years, showed a significant reduction (35\%) in deaths due to cardiovascular disease and nonfatal infarction in patients undergoing partial ileal bypass surgery. The population assessed in this study did not consist of patients with familial hypercholesterolemia; the mean total cholesterol at admission was $251 \mathrm{mg} / \mathrm{dL}$ and the mean LDL-C was $179 \mathrm{mg} / \mathrm{dL}$. These values did not indicate therapeutic resistance to lipid-lowering drugs, and, with the measures currently available (statins), significant reductions in cholesterolemia may be obtained with no need for invasive procedures. At any rate, the findings regarding morbidity and mortality are consistent and show that this procedure allows a persistent reduction in cholesterolemia with a decrease in cholesterol levels in patients with coronary artery disease.

On the other hand, the experience with partial ileal bypass surgery in the treatment of patients with heterozygous familial hypercholesterolemia shows that a $33 \%$ reduction in cholesterolemia occurs associated with a reduction in cardiovascular morbidity and mortality after a 10-year follow-up ${ }^{7}$, despite the persistence of high levels of cholesterolemia (total cholesterol prior to thepartial ileal bypass surgery, $530 \mathrm{mg} /$ $\mathrm{dL}$; total cholesterol after the procedure, $356 \mathrm{mg} / \mathrm{dL}$ ).

In our patients undergoing partial ileal bypass surgery, a persistent $30 \%$ to $35 \%$ reduction in total cholesterol occurred, making the significant regression of the xanthomas and also the reduction in the symptoms of coronary atherosclerotic disease possible, despite the still high cholesterolemia. Thompson et al ${ }^{8}$ report that the significant percentage reduction in cholesterolemia is enough to improve the prognosis of the coronary atherosclerotic disease, independently from the serum levels of total cholesterol obtained ${ }^{8}$.

By means of comparison, we assessed the evolution of another patient, a 43-year-old male sibling of the patients in cases 1 and 2, also with xanthomatous hypercholesterolemia (total cholesterol of $900 \mathrm{mg} / \mathrm{dL}$ ), coronary heart disease 


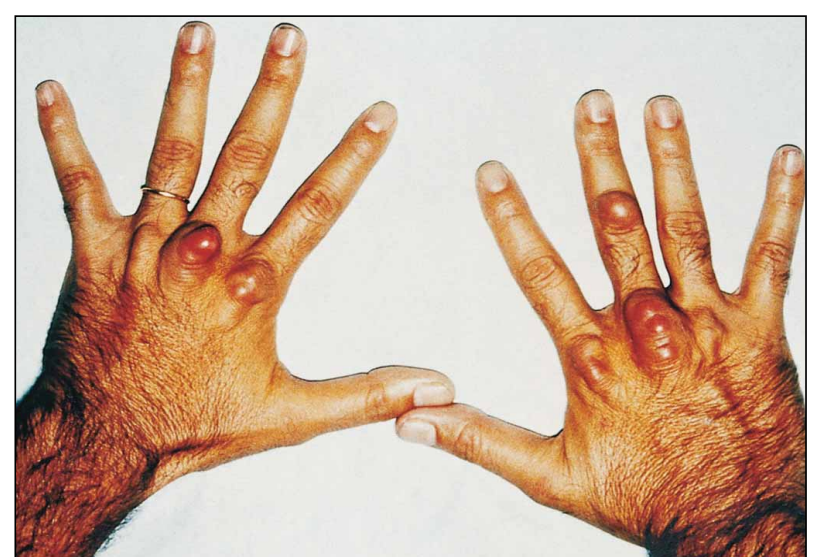

Fig. 7 - Tendinous xanthomas in the hands of the patient sibling of cases 1 and 2 who refused to undergo the ileal bypass surgery.

and aortic stenosis. He underwent myocardial revascularization and placement of an aortic metallic prosthesis in 1990 in our hospital. He refused to undergo the partial ileal bypass surgery in 1991, and in the same period of follow-up (8 years) of his sisters, during which he irregularly used statins and probucol, his xanthomas progressed (figs. 7 and 8).

We conclude that partial ileal bypass surgery, in spite of being invasive and having side effects, is a reversible procedure that may be a therapeutic option for patients with severe hypercholesterolemia and coronary heart disease, given the high cost of the medicamentous therapy for a significant part

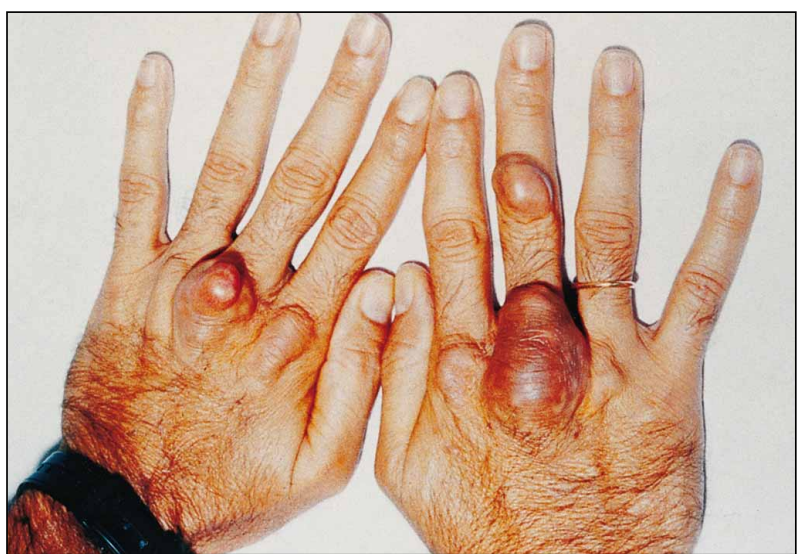

Fig. 8 - Same patient of fig. 7 evolution of the xanthomas after a 6-year follow-up with irregular use of lipid-lowering drugs ( Same patient of fig. 7).

of the Brazilian population. We think this procedure is a therapeutic option even considering the recent possibility of using more potent statins, such as atorvastatin, and higher doses of other statins (e.g. simvastation).

Having said this, we understand that young patients with familial hypercholesterolemia and coronary atherosclerotic disease may benefit from partial ileal bypass surgery. We would like to suggest that this procedure should be considered in referral centers that include a service specializing in dyslipidemia and a surgical team with experience in similar procedures.

\section{References}

1. Goldstein JL, Brown MS. The LDL-receptor defect in familial hypercholesterolemia. Implications for pathogenesis and therapy. Med Clin North Am 1982; 66 : 335-62.

2. Avila WS, Issa JS, Bezi EB, et al. Gestação em portadora de hipercolesterolemia familiar e doença aterosclerótica prematura. Arq Bras Cardiol 1997; 68: 185-8.

3. Issa JS, Safi Jr J. Dislipidemias graves: medidas alternativas. Rev Bras Med 1998 55 (Ed. especial ): 29-34.

4. Buchwald $\mathrm{H}$. Lowering of cholesterol absorption and blood levels by ileal exclusion: experimental basis and preliminary clinical report. Circulation 1964; 29:713-20.

5. Buchwald H, Varco R, Matts JP, et al. Effect of partial ileal bypass surgery on mortality and morbidity from coronary heart disease in patients with hypercholesterolemia. N Engl J Med 1990, 323: 946.

6. Buchwald H, Varco RL, Boen JR, et al. Effective lipid modification by partial ileal bypass reduced long-term coronary heart disease mortality and morbidity: fiveyears postrial follow-up report from the POSCH. Program on the Surgical Control of the Hyperlipidemias. Arch Intern Med 1998; 158: 1253-61.

7. Koivisto P, Miettinen TA. Long-term effects of ileal bypass on lipoproteins in patients with familial hypercholesterolemia. Circulation 1984; 70: 290-6.

8. Thompson GR, Hollyer J, Waters DP. Percentage change rather than plasma level of LDL-Cholesterol determines therapeutic response in coronary heart disease. Current Opin Lipidol 1995; 6: 386-8. 